\title{
Modified Severity Weighted Assessment Tool
}

National Cancer Institute

\section{Source}

National Cancer Institute. Modified Severity Weighted Assessment Tool. NCI Thesaurus. Code C159687.

A clinical measurement tool for measuring skin tumor burden in mycosis fungoides (MF) and Sezary syndrome (SS). It involves determining the percentage of body surface area (BSA) covered by patch, plaque, or tumor of MF/SS, then multiplying each lesion BSA by a factor that gives gradations of weight to patch versus plaque versus tumor and summing these scores. The change in mSWAT from the beginning of treatment can be used to assess overall response. 